PROCEEDINGS OF THE

AMERICAN MATHEMATICAL SOCIETY

Volume 136, Number 8, August 2008, Pages 2875-2879

S 0002-9939(08)09201-0

Article electronically published on April 8, 2008

\title{
ASCOLI-ARZELÀ TYPE THEOREM \\ FOR RATIONAL ITERATIONS ON THE COMPLEX PROJECTIVE PLANE
}

\author{
KAZUTOSHI MAEGAWA
}

(Communicated by Mei-Chi Shaw)

\begin{abstract}
For a dominant algebraically stable rational self-map of the complex projective plane of degree at least 2 , we will consider three different definitions of the Fatou set and show the equivalence of them (Ascoli-Arzelà type theorem). As a corollary, it follows that all Fatou components are Stein. This is an improvement of an early result by Fornæss and Sibony.
\end{abstract}

\section{INTRODUCTION}

The study of complex dynamics in higher dimensions has been developed intensively, but some early papers such as [FS are still helpful for recent studies. In those papers, the dynamics of rational self-maps of the complex projective spaces are studied, and several fundamental theorems concerning the Fatou set and the Julia set are contained there.

In this paper, we will improve two theorems which are contained in FS] (Theorem 5.2 and Theorem 5.7). When we compare rational maps of $\mathbb{P}^{2}$ with those of $\mathbb{P}^{1}$, one of the remarkable differences is that those of $\mathbb{P}^{2}$ possibly have indeterminacy points. If we want to generalize the Fatou-Julia theory to dimension two, we have to find a suitable way to define the Fatou set and the Julia set paying attention to indeterminacy points. So, in this paper, for an algebraically stable rational self-map $f$ of $\mathbb{P}^{2}$ of degree at least 2, we will consider several different definitions of the Fatou set and show the equivalence of them (Ascoli-Arzelà type theorem). One of them is the standard; that is, it is based on local equicontinuity of the iterates of $f$, i.e. Lyapunov stability. The others are more complex analytical. They are based on notions of normal family for meromorphic maps. Throughout this paper, we often use Ivashkovich's theorems in [I] and Fornæss-Sibony's theorems concerning Green currents in $[\underline{\mathrm{S}}$. Particularly, by using Ivashkovich's result on domains of normality for families of meromorphic maps, we can show that all Fatou components for $f$ are Stein manifolds.

Received by the editors October 24, 2006, and, in revised form, April 17, 2007.

2000 Mathematics Subject Classification. Primary 32H50; Secondary 32Q28.

Key words and phrases. Rational maps, Fatou sets, Stein manifolds. 


\section{Preliminaries}

We denote by $\mathbb{P}^{k}$ the complex projective space of complex dimension $k \geq 1$ and we equip the Fubini-Study distance with $\mathbb{P}^{k}$. By definition, there is a projection

$$
\pi: \mathbb{C}^{k+1} \backslash\{O\} \rightarrow \mathbb{P}^{k}
$$

which is a $\mathbb{C}^{*}$-bundle over $\mathbb{P}^{k}$, where $O$ denotes the origin of $\mathbb{C}^{k+1}$. By definition, a rational self-map $f$ of $\mathbb{P}^{k}$ is lifted by $\pi$ to a polynomial self-map $\bar{f}$ of $\mathbb{C}^{k+1}$ which is of the form

$$
\bar{f}=\left(P_{0}, \cdots, P_{k}\right)
$$

where $P_{i}, 0 \leq i \leq k$, are homogeneous polynomials which have the same degree and have no common factors. The degree $\operatorname{deg}(f)$ of $f$ is defined to be the degree of $P_{i}$. A point $p \in \mathbb{P}^{k}$ such that $\bar{f}\left(\pi^{-1}(p)\right)=O$ is an indeterminacy point for $f$. We denote by $I=I(f)$ the set of indeterminacy points for $f$. The dimension of $I$ is at most $k-2$ if $k \geq 2$. (If $k=1$, then $I$ is empty.) We obtain the lift of the $n$-th iterate $f^{n}=f \circ \cdots \circ f$ ( $n$ times) by the cancellation of common factors of the component functions for $\bar{f}^{n}$.

Definition $2.1([\underline{S}])$. We say that $f$ is algebraically stable (AS for short) if $f^{-1}\left(I\left(f^{n}\right)\right)$ contains no complex hypersurface in $\mathbb{P}^{k}$ for all $n \geq 1$. This is equivalent to the condition that $\operatorname{deg}\left(f^{n}\right)=(\operatorname{deg}(f))^{n}$ for all $n \geq 1$. (In other words, for all $n \geq 1$, there are no common factors of the component functions for $\bar{f}^{n}$.)

Remark 2.2. In the case when $f$ is $\mathrm{AS}, I\left(f^{n}\right)=\pi\left(\bar{f}^{-n}(O)\right)$ for all $n \geq 1$. In particular, $I\left(f^{n}\right) \subset I\left(f^{n+1}\right)$ for all $n \geq 1$ because $\bar{f}(O)=O$.

Let $\omega$ denote the normalized Fubini-Study $(1,1)$ form in $\mathbb{P}^{k}$ and let $f$ be an AS rational self-map of $\mathbb{P}^{k}$ of degree $d \geq 2$ which is dominant, i.e. $f\left(\mathbb{P}^{k}\right)=\mathbb{P}^{k}$. Then,

$$
\frac{1}{d^{n}}\left(f^{n}\right)^{*} \omega \rightarrow T
$$

as $n \rightarrow \infty$ in the sense of currents, where $T$ is a positive closed $(1,1)$ current such that $f^{*}(T)=d T(\underline{\underline{S}})$. We call $T$ the Green $(1,1)$ current for $f$. The current $T$ is of the form

$$
T=\omega+\mathrm{dd}^{\mathrm{c}} v
$$

where $v$ is an integrable function in $\mathbb{P}^{k}$.

Let us note that for any $m \geq 1$, the map $f^{m}$ is holomorphic in the complement of the closure of $\bigcup_{n \geq 1} I\left(f^{n}\right)$.

Definition 2.3. We define the Fatou set $F$ to be the maximal open subset of $\mathbb{P}^{k} \backslash \overline{\bigcup_{n \geq 1} I\left(f^{n}\right)}$ on which $\left\{f^{n}\right\}$ is locally equicontinuous. A connected component of $F$ is called a Fatou component. The complement $J$ of $F$ is called the Julia set for $f$.

The support of $T$ is extremely related to $J$. In particular, the following equality is known.

Theorem 2.4 ([FS, $[\mathrm{U}])$. In the case when $f$ is holomorphic, $J=\operatorname{supp}(T)$. 


\section{RESUlts}

Here we will show our theorem. Let us begin with preparing some notions.

Definition 3.1 (II $]$ ). Let $X, Y$ be complex manifolds. Let $\left\{g_{n}\right\}_{n \geq 1}$ be a sequence of meromorphic maps from $X$ to $Y$. Let $\Gamma_{n} \subset X \times Y$ denote the graph of $g_{n}$. Let $g: X \rightarrow Y$ be a meromorphic map and let $\Gamma \subset X \times Y$ denote the graph of $g$.

(i) We say that $\left\{g_{n}\right\}_{n \geq 1}$ strongly converges to $g$ in $X$ if for any compact set $K \subset X$,

$$
\lim _{n \rightarrow \infty} \Gamma_{n} \cap(K \times Y)=\Gamma \cap(K \times Y)
$$

with respect to the Hausdorff metric.

(ii) We say that $\left\{g_{n}\right\}_{n \geq 1}$ weakly converges to $g$ in $X$ if there is an analytic subset $A \subset X$ of $\operatorname{codim}_{\mathbb{C}} A \geq 2$ such that $\left\{g_{n}\right\}_{n \geq 1}$ strongly converges to $g$ in $X \backslash A$.

Remark 3.2. The definition of strong convergence above is slightly modified from the original one in [I]. It is only a name change.

By using these two notions of convergence, we can introduce notions of normality for a sequence of meromorphic maps in the strong and weak senses; that is, we say that a sequence $\left\{f_{n}\right\}$ of meromorphic maps from $X$ to $Y$ is strongly (resp. weakly) normal if for any subsequence $\left\{f_{n_{j}}\right\}$ of $\left\{f_{n}\right\}$, there is a subsequence of $\left\{f_{n_{j}}\right\}$ which converges in $X$ in the strong (resp. weak) sense. Thus, for the iterates of a rational self-map of $\mathbb{P}^{2}$, we can define the strong (resp. weak) Fatou set $F_{s}$ (resp. $F_{w}$ ) as the maximal open subset of $\mathbb{P}^{2}$ on which the iterates is strongly (resp. weakly) normal.

Now we can state our main theorem:

Theorem 3.3 (Ascoli-Arzelà type theorem). Let $f$ be a dominant AS rational self-map of $\mathbb{P}^{2}$ of degree at least 2 . Then,

$$
F=F_{s}=F_{w} .
$$

To show this, we need the following theorems, which are contained in Ivashkovich's paper [I].

Theorem $3.4\left([\mathbb{I})\right.$. Let $f$ be a rational self-map of $\mathbb{P}^{2}$. Then, the following (i) and (ii) hold.

(i) $F_{w}$ is pseudoconvex.

(ii) If $F_{s} \neq F_{w}$, then $F_{w} \supset \mathbb{P}^{2} \backslash C$, where $C$ is a rational curve in $\mathbb{P}^{2}$.

Theorem 3.5 (II] (Rouché Principle)). Let $X, Y$ be complex manifolds. Let $f_{n}$ : $X \rightarrow Y, n \geq 1$, be meromorphic maps which strongly converge in $X$ to a meromorphic map $f: X \rightarrow Y$ as $n \rightarrow \infty$. Then, the following $(a)$ and (b) hold.

(a) If $f$ is holomorphic, then for any relatively compact open subset $D$ in $X$, the restrictions $f_{n} \mid D$ for sufficiently large $n$ are holomorphic in $D$ and $\left\{f_{n}\right\}$ converges to $f$ uniformly on compact sets in $X$.

(b) If all $f_{n}$ are holomorphic, then $f$ is also holomorphic and $\left\{f_{n}\right\}$ converges uniformly on compact sets in $X$.

We also need the following two theorems, which come from the study of dynamics using pluripotential theory. In particular, Theorem 3.8 will play a crucial role.

Theorem $3.6(\underline{\underline{S}}])$. Let $T$ be the Green $(1,1)$ current for a dominant AS rational self-map $f$ of $\mathbb{P}^{k}$ of degree at least 2 . Then, $F \subset \mathbb{P}^{k} \backslash \operatorname{supp}(T)$. 
Remark 3.7. We can easily obtain a slightly modified version of this. That is, if a subsequence $\left\{f^{n_{j}}\right\}_{j \geq 1}$ converges uniformly in an open set $U$ in $\mathbb{P}^{k}$, then $U \subset$ $\mathbb{P}^{k} \backslash \operatorname{supp}(T)$.

Let $K$ be a closed set in $\mathbb{P}^{k}$ and let $S$ be a positive current in $\mathbb{P}^{k} \backslash K$ with locally bounded mass near $K$. The trivial extension of $S$ to $\mathbb{P}^{k}$ is obtained by setting the coefficients of $S$ to be 0 on $K$. (Note that the coefficients of $S$ are complex measures.)

Theorem $3.8([\underline{\mathrm{S}})$. Let $T$ be the Green $(1,1)$ current for a dominant AS rational self-map $f$ of $\mathbb{P}^{k}$ of degree at least 2 . Then, $T$ does not charge on any complex hypersurface $V$; i.e., $T$ is equal to the trivial extension of $\left.T\right|_{\mathbb{P}^{k} \backslash V}$.

Proof of Theorem 3.3. By definition, it is easily shown that

$$
F \subset F_{s} \subset F_{w} \text {. }
$$

Let us show that $F_{s}=F_{w}$. Let $U_{w}$ be a connected component of $F_{w}$. Let $\left\{f^{n_{k}}\right\}_{k \geq 1}$ be any subsequence of $\left\{f^{n}\right\}_{n \geq 1}$ which weakly converges in $U_{w}$. Then, there is a discrete set $A \subset U_{w}$ such that $\left\{f^{n_{k}}\right\}_{k \geq 1}$ strongly converges to a meromorphic map $\psi$ in $U_{w} \backslash A$. Let $I(\psi)$ denote the indeterminacy set of $\psi$. By definition, $\psi$ is holomorphic in $U_{w} \backslash I(\psi)$. Suppose that there is $m \geq 0$ such that $f^{m}$ is not holomorphic at $p \in U_{w} \backslash(I(\psi) \cup A)$. From Remark 2.2, it follows that $f^{n}$ is not holomorphic at $p$ for $\forall n \geq m$. By the Rouché principle (Theorem 3.5), $\psi$ is also not holomorphic at $p$. This is a contradiction. So, $f^{n}$ are holomorphic in $U_{w} \backslash(I(\psi) \cup A)$ for all $n \geq 1$. Further, $\left\{f^{n_{k}}\right\}_{k \geq 1}$ converges locally uniformly to $\psi$ in $U_{w} \backslash(I(\psi) \cup A)$. By Remark 3.7, it follows that $U_{w} \backslash(I(\psi) \cup A)$ is contained in $\mathbb{P}^{2} \backslash \operatorname{supp}(T)$. Since $I(\psi) \cup A$ is discrete, the local potential function for $T$ should be pluriharmonic in $U_{w}$. Hence, it follows that $U_{w} \subset \mathbb{P}^{2} \backslash \operatorname{supp}(T)$. Thus, $F_{w} \subset \mathbb{P}^{2} \backslash \operatorname{supp}(T)$. Suppose that $F_{s} \neq F_{w}$. By (ii) in Theorem 3.4. $F_{w} \supset \mathbb{P}^{2} \backslash C$, where $C$ is a rational curve. Hence, $C \supset \operatorname{supp}(T)$. However this is impossible because $T$ does not charge on $C$ (Theorem 3.8). Thus, it follows that $F_{s}=F_{w}$.

Let us show that $F=F_{s}$. Let $U_{s}$ be a connected component of $F_{s}$. Let $\left\{f^{n_{k}}\right\}_{k \geq 1}$ be any subsequence of $\left\{f^{n}\right\}_{n \geq 1}$ which strongly converges in $U_{s}$. Denote the limit map by $\phi$ and let $I(\phi)$ be the indeterminacy set for $\phi$. In the same way as we have done above, it follows that $U_{s} \subset \mathbb{P}^{2} \backslash \operatorname{supp}(T)$. Suppose that $I(\phi)$ is nonempty and take $q \in I(\phi)$. By the Rouché principle and Remark 2.2, there is an integer $m \geq 0$ such that $f^{m}(q) \in I(f)$. So, it follows that $q \in \operatorname{supp}(T)$ since the Lelong number for $T$ at $q$ must be strictly positive (this can be shown by the assumption that $f$ is AS). This is a contradiction to $U_{s} \subset \mathbb{P}^{2} \backslash \operatorname{supp}(T)$. Hence, $I(\phi)=\emptyset$. Again by the Rouché principle, it follows that $\left\{f^{n_{k}}\right\}_{k \geq 1}$ are holomorphic in $U_{s}$ and converge locally uniformly to $\phi$ in $U_{s}$. This leads to $U_{s} \subset F$. Hence, $F_{s} \subset F$.

Thus,

$$
F=F_{s}=F_{w}
$$

are verified.

Corollary 3.9. Let $f$ be a dominant AS rational self-map of $\mathbb{P}^{2}$ of degree at least 2. Then, any Fatou component for $f$ is Stein.

Proof. By (i) in Theorem 3.4 and the equalities in Theorem 3.3 , it follows that $F$ is pseudoconvex. It is known that any pseudoconvex domain in $\mathbb{P}^{k}$, which is not $\mathbb{P}^{k}$, is Stein (see [T]). So, it follows that $F$ is Stein. 
Remark 3.10. It is known that $F \subset \mathbb{P}^{2} \backslash \operatorname{supp}(T)$ (Theorem 3.6). However, we cannot expect the equality because there is an example for which $F \neq \mathbb{P}^{2} \backslash \operatorname{supp}(T)$. See Example 2 in $[\mathrm{RS}$.

As a consequence of Corollary 3.9, the connectivity of the Julia set follows. It is a common property of pseudoconcave sets in $\mathbb{P}^{k}$.

Corollary 3.11. Let $f$ be a dominant $A S$ rational self-map of $\mathbb{P}^{2}$ of degree at least 2. Then, the Julia set $J$ is connected.

Proof. The proof is valid in the same way as in Theorem 5.2 in [FS].

Let us note that Theorem 3.3 does not necessarily hold in the case of non-AS maps. An example is a birational map $f([x: y: z])=[y z: z x: x y]$. When $m$ is even, $f^{m}$ is the identity map, and when $m$ is odd, $f^{m}=f$. Hence, it follows immediately that $F_{s}=F_{w}=\mathbb{P}^{2}$. However, since $f$ has indeterminacy points $[1: 0$ : $0],[0: 1: 0],[0: 0: 1]$, the set $F$ is smaller than $\mathbb{P}^{2}$. In fact, $F=\mathbb{P}^{2} \backslash \pi(\{x y z=0\})$.

In this paper, we dealt only with $\mathrm{AS}$ rational maps on $\mathbb{P}^{2}$. It seems interesting to extend our theorem to some meromorphic maps on the other surfaces (manifolds).

\section{REFERENCES}

[FS] J. E. Fornæss And N. Sibony, Complex dynamics in higher dimension II. Ann. of Math. Stud., 137, Princeton University Press, 1995, 134-182. MR1369137 (97g:32033)

[I] S. IvashKovich, On convergence properties of meromorphic functions and mappings. (Russian), Complex analysis in modern mathematics, FAZIS, Moscow, 2001, 133-151. English manuscript available at ArXiv math.CV/9804007. MR.1833510(2002e:32023)

[RS] A. Russakovskit and B. Shiffman, Value distribution for sequences of rational mappings and complex dynamics. Indiana Univ. Math. J., 46 (1997), No. 3, 897-932. MR1488341 (98h:32046)

[S] N. Sibony, Dynamique des applications rationnelles de $\mathbb{P}^{k}$. Panor. Synthèses, 8, Soc. Math. France, 1999, 97-185. MR1760844 (2001e:32026)

[T] A. TAKeuchi, Domaines pseudoconvexes infinis et la métrique riemannienne dans un espace projectif. J. Math. Soc. Japan, 16 (1964), 159-181. MR0173789(30:3997)

[U] T. UedA, Fatou sets in complex dynamics on projective spaces. J. Math. Soc. Japan, 46 (1994), no. 3, 545-555. MR.1276837 (95d:32030)

Department of Mathematics, Graduate School of Science, Kyoto University, Kyoto, 606-8502, JAPAN

E-mail address: maegawa@math.kyoto-u.ac.jp 